\title{
Quadratic Fields with Four Invariants Divisible by 3
}

\author{
By Daniel Shanks and Richard Serafin
}

\begin{abstract}
Imaginary quadratic fields are developed that have four invariants divisible by 3. Their associated real fields are found to differ in one significant respect: one case has two elementary generators and the other has only one.
\end{abstract}

1. Series 6. The number of invariants of a quadratic field $Q\left(d^{1 / 2}\right)$ that are divisible by 3 equals the number of factors in the 3-Sylow subgroup of its class group. Following Scholz [1], we use $r$ for this number if $d<0$ and $s$ if $d>0$. The first case of $r=1$ is $Q\left((-23)^{1 / 2}\right)$. This has $C(3)$ as its class group. The first case [2] of $r=2$ is $Q\left((-3299)^{1 / 2}\right)$ with $C(9) \times C(3)$. The smallest known case [3] of $r=3$ is $Q\left((-63199139)^{1 / 2}\right)$ with $C(3) \times C(3) \times C(3) \times C(116)$. These three discriminants are

$$
-D_{6}(1), \quad-D_{6}(-2), \quad-D_{6}(28)
$$

respectively, where

$$
D_{6}(z)=108 z^{4}-148 z^{3}+84 z^{2}-24 z+3 .
$$

It was proven in [3] that $r \geqq 2$ for all square-free discriminants $-D_{6}(z)$ with $z \equiv 1(\bmod 3)$ except for the degenerate $z=1$. It was also shown that $r=3$ for

$$
z=28,-29, \quad 34,-41,-44,46,
$$

and while it was not proven that $-D_{6}(z)$ yields infinitely many cases of $r>2$, that seemed very probable. If one continues (2), one finds that $r=3$ also for

$$
z=79,-92,-122,-125,127,-131,148,-164 .
$$

Empirically, about $1 / 6$ of all square-free $D_{6}(z)$ have $r>2$ and it seemed plausible [3] that after a moderate number of such $r>2$ were located, an example of $r=4$ would appear. But this was not pursued at the time.

Recently, we learned from Professor D. J. Lewis that a doctoral student of his, Maurice Craig [4], had constructed a $Q\left((-D)^{1 / 2}\right)$ with $r=4$. No details were conveyed except that $D$ is very large, of the order of $400 \cdot 10^{100}$, and so it is not suitable for a detailed numerical examination. To prove the existence of an $r=4$, only one case is needed, but, analytically speaking, some interest attaches to the size of the smallest such $D$. Thus, we could ask: How big must $D$ be for the Diophantine equation

$$
4 a^{3}=b^{2}+c^{2} D
$$

to have 81 distinct solutions with $0<a<(D / 3)^{1 / 2}, 0<b,(b, c) \leqq 2$ ? Such solutions

Received May 15, 1972.

AMS (MOS) subject classifications (1970). 12A25, 12A50.

Copyright $\odot$ 1973, American Mathematical Society 
correspond to ideals $\mathfrak{A}=\left(a,\left(b+c(-D)^{1 / 2}\right) / 2\right)$ whose cube is principal:

$$
\mathfrak{A}^{3}=\left(\frac{b+c(-D)^{1 / 2}}{2}\right) \text {. }
$$

Since it appeared likely that a much smaller $D$ could be obtained with $D_{6}(z)$, we therefore continued (2) and found that the next case after (2a) does have $r=4$. This is

$$
D=D_{6}(169)=87386945207=167 \cdot 12409 \cdot 42169
$$

which has the class group

$$
C(3) \times C(3) \times C(3) \times C(3) \times C(1448) \times C(2) .
$$

To verify that $[C(3)]^{4}$ is a subgroup, it suffices to verify the 14 solutions of (3) in Table 1.

\section{TABLE 1}

\begin{tabular}{rrrl}
\multicolumn{1}{c}{$a$} & \multicolumn{1}{c}{$b$} & \multicolumn{1}{c}{$c$} & Structure \\
113738 & 76715859 & 1 & $J$ \\
6854 & 1095693 & -1 & $K$ \\
89158 & 40480625 & 117 & $J^{2} K$ \\
11904 & 2580707 & 1 & $J^{2} K^{2}$ \\
22574 & 6776883 & 1 & $L$ \\
106028 & 65782389 & 71 & $J^{2} L$ \\
164511 & 133418432 & 10 & $J L$ \\
112456 & 2509283 & 255 & $K^{2} L^{2}$ \\
73278 & 18341941 & -119 & $K L^{2}$ \\
96774 & 20911027 & -191 & $J^{2} K^{2} L^{2}$ \\
11321 & 459414 & -8 & $J K^{2} L^{2}$ \\
31972 & 8186767 & -27 & $J^{2} K L^{2}$ \\
131167 & 38385160 & 294 & $J K L^{2}$ \\
2802 & 24685 & 1 & $M$
\end{tabular}

These 14, together with their 14 inverses obtained by changing the sign of $c$, correspond to 28 ideals of order 3 and minimal norm $a$ within their respective equivalence classes. Since the identity of a class group with $r<4$ can have at most 27 such cube-roots, we must have $r \geqq 4$.

The entries $J, K, L, M$ constitute four generators and the products of the first three make up the other rows in Table 1 and their inverses: $J^{2}, K^{2}, J K^{2}$, etc. $J$ is the "elementary explicit cube-root" [3] given by

$$
a=4 z^{2}-3 z+1, \quad b=16 z^{3}-18 z^{2}+6 z-1, \quad c=1 .
$$

The remaining 26 values of $a$ are obtained by taking all ideal products with $M$. They are, in order of size, 3378, 4208, $\cdots$, 156228. All 40 values of $a$ are distinct. The four generators could have been selected in 24261120 ways; e.g., in place of the $K$ and $L$ shown we could have taken the smaller 3378 and 4208 , both of which also have $c=1$. 
By Scholz's theorem [1] and Theorem 3 of [3], the real field $Q\left(\mathcal{V} 3 D_{6}(169)\right)$ will have $s=3$. Its group is

$$
C(9) \times C(3) \times C(3) \times C(16) \times C(2) .
$$

Since its class number $h=2592$ is relatively large for a real field, its fundamental unit $\epsilon=\left(T+U(3 D)^{1 / 2}\right) / 2$ is correspondingly not too large to be given exactly:

$$
\begin{array}{rr}
T=96179600759735540636573164931915352034585, \\
U= & 187844750873014264050654697450227699 .
\end{array}
$$

It is desirable to explain how the $r=4$ here comes about. In [3], it is proven that $r=s+1$ for $-D_{6}(z)$ and $3 D_{6}(z), z \equiv 1(\bmod 3)$, and of the two solutions of

$$
4 a^{3}=b^{2}-c^{2} 3 D
$$

given by

$$
\begin{array}{llrl}
a=3 z, & b=54 z^{2}-36 z+9, & c=3, \\
a=3 z-2, & b=54 z^{2}-36 z+7, & c=3,
\end{array}
$$

at least one corresponds to an ideal of order 3 in $Q\left((3 D)^{1 / 2}\right)$. Then $s=2$ and $r=3$ will occur if

(1) both ideals $(6 a, b)$ are of order 3 and independent, or

(2) a third ideal, independent of (6a) and (6b), is of order 3.

Both possibilities happen. Then, as predicted in [5, p. 86], if both (1) and (2) occur we will have $s=3$ and $r=4$. This happens for $z=169$ with the fourth power of a prime ideal of norm 5 . The prime ideal is of order 12 , and its fourth power is a third, independent generator. Owing to the size of $\epsilon$, its $b$ and $c$ are large:

$$
\begin{array}{lr}
a= & 625, \\
b & =12281994225220152913, \\
c & =23987499711333 .
\end{array}
$$

Continuing $D_{6}(z)$ for a few more values of $z$ (to comprise exactly 100 discriminants) yields two more examples of $r=3$ at $z=-170$ and $z=175$.

2. Series 3. Series 3 are [3] the square-free

$$
D_{3}(y)=27 y^{4}-74 y^{3}+84 y^{2}-48 y+12
$$

with $y \equiv-1(\bmod 6)$. We did not similarly extend the earlier table of $D_{3}(y)$ by examining each successive case; we confined ourselves to selected $D_{3}(y)$ that are either prime or, on the contrary, have many factors. Thus,

$$
D=D_{3}(-235)=83309629817 \equiv 1 \quad(\bmod 4)
$$

is prime and the class group of $Q\left((-D)^{1 / 2}\right)$ is

$$
C(9) \times C(3) \times C(3) \times C(3) \times C(724)
$$

with $r=4$. The 40 inequivalent ideals $\left(a, b+c(-D)^{1 / 2}\right)$ satisfying $a^{3}=b^{2}+c^{2} D$ are listed in Table 2. 
TABLE 2

\begin{tabular}{rrrrrr}
\multicolumn{7}{c}{$a^{3}=b^{2}+c^{2} 83309629817$} \\
\multicolumn{1}{c}{$b$} & \multicolumn{1}{c}{$b$} & \multicolumn{1}{c}{$c$} & \multicolumn{1}{c}{$a$} & \multicolumn{1}{c}{$c$} \\
\hline 6957 & 58985 & 2 & 140317 & 50197121 & 54 \\
7629 & 332839 & 2 & 150421 & 6140438 & 201 \\
7898 & 399282 & 2 & 176538 & 72976990 & 46 \\
9218 & 670842 & 2 & 181157 & 48022254 & 209 \\
11714 & 1128774 & 2 & 193773 & 28935833 & 278 \\
16258 & 1139390 & 6 & 194338 & 84241810 & 54 \\
45482 & 9682530 & 2 & 204369 & 2215897 & 320 \\
47381 & 7368846 & 25 & 222314 & 104819874 & 2 \\
47441 & 9243687 & 16 & 226409 & 101197899 & 128 \\
63029 & 15813261 & 2 & 232261 & 37007227 & 366 \\
78282 & 21894850 & 2 & 234981 & 6486427 & 394 \\
84033 & 20787385 & 44 & 237546 & 90539594 & 250 \\
86074 & 11438686 & 78 & 238474 & 9940366 & 402 \\
95317 & 27189566 & 39 & 249026 & 101174274 & 250 \\
100938 & 21638642 & 82 & 265301 & 51162438 & 439 \\
101194 & 29820986 & 42 & 265554 & 135345358 & 70 \\
107241 & 34813963 & 16 & 277818 & 49070002 & 478 \\
120889 & 41889061 & 12 & 293458 & 145485622 & 222 \\
137058 & 42877690 & 94 & 297309 & 155648414 & 157 \\
137673 & 35277317 & 128 & 302241 & 150498103 & 244
\end{tabular}

Both (4) and (9) contain $C(181)$. Presumably, this is a coincidence; if it had some causal significance that would certainly be of interest! Also puzzling are the pairs with $c=c_{1}$ or $c=2 c_{1}$. See $c=78,16,12,54,128,402,250$ in Table 2.

The class group of $Q\left((3 D)^{1 / 2}\right)$ is now $C(3) \times C(3) \times C(3) \times C(2)$. The elementary solutions of

$$
a^{3}=b^{2}-c^{2} 3 D
$$

are

(10a)

$$
\begin{array}{llrl}
\text { (10a) } & a=6 y, & b=54 y^{2}-72 y+36, & c=6, \\
\text { (10b) } & a=6 y-8, & b=54 y^{2}-72 y+28, & c=6,
\end{array}
$$

for Series 3 but for $y=-235$ the ideal corresponding to (10a) is now principal. So for $D_{3}(-235)$ there are two additional ideals of order 3 that are independent of $(10 a, b)$ and each other. The ideal for $(10 b)$ is equivalent to a prime ideal of norm 37 and the two other generators can be taken as prime ideals of norm 23 and 71 . We may therefore escalate our expectations and now expect cases with $s=4$ and $r=5$.

On a point of terminology that frequently causes confusion: When we wrote that the first case of $r=2$ is $Q\left((-3299)^{1 / 2}\right)$, we meant that 3299 is the minimal absolute value of the discriminant. As is known, $Q\left((-D)^{1 / 2}\right)$ also has $r=2$ for $D=974$ and 2437 , but here the discriminant is $-4 D$, not $-D$. Of course, "first case" and 
"smallest" can equally well be defined to mean the smallest $D$, and some well-known books assert that $Q\left((-5)^{1 / 2}\right)$ is the first case of nonunique factorization while others say that $Q\left((-15)^{1 / 2}\right)$ is. By our choice, $Q\left(\left(-D_{6}(169)\right)^{1 / 2}\right)$ is the "smaller" of our two cases of $r=4$ even though (8) is smaller than (2b). That seems the preferred convention in this context; e.g., compare the values of $a$ in Tables 1 and 2 .

Finally, since it may be of interest, we record

$$
D=D_{3}(449)=1090678524545=5 \cdot 23 \cdot 83 \cdot 193 \cdot 592057 .
$$

Here, $Q\left((-D)^{1 / 2}\right)$ has (only) $r=3$ but the 2-Sylow subgroup has five factors in addition:

$$
C(9) \times C(3) \times C(3) \times C(8) \times C(2) \times C(2) \times C(2) \times C(2) \times C(73) .
$$

3. The Class Field Towers. Golod and Safarevic proved [6] that the class field tower of an algebraic field $k$ is infinite if its class group requires sufficiently many generators. Such $k$ therefore cannot be imbedded in a larger algebraic field, of finite degree, having unique factorization. Specifically, from Roquette's formula [7, Eq. (1), p. 233], it follows that an imaginary quadratic field does have an infinite tower if its 3-rank (our $r$ above) exceeds 3 . So $Q\left((-D)^{1 / 2}\right.$ ) has such a tower for the $D$ in (2b) and (8), the second case being especially noteworthy since its $D$ is prime. The $Q\left((-D)^{1 / 2}\right)$ for (11) has an infinite tower because of its 2-rank = 5 (see Roquette, p. 234), but whether its 3 -rank $=3$ would also suffice is apparently not now known.

Computation \& Mathematics Department

Naval Ship Research \& Development Center

Bethesda, Maryland 20034

1. A. Scholz, "Uber die Beziehung der Klassenzahlen quadratischer Körper zueinander," Crelle's J., v. 166, 1932, pp. 201-203.

2. A. Scholz \& OLgA TAUSSKY, "Die Hauptideale der kubischen Klassenkörper imaginär quadratischer Zahlkörper: ihre rechnerische Bestimmung und ihr Einfluss auf den Klassenkörperturm," Crelle's J., v. 171, 1934, pp. 19-41.

3. DANIEl SHANKS, "New types of quadratic fields having three invariants divisible by 3 ," J. Number Theory. (To appear.)

4. Maurice CRAIG, Irregular Discriminants, Dissertation, University of Michigan, Ann Arbor, Mich., 1972.

5. Daniel Shanks \& Peter Weinberger, "A quadratic field of prime discriminant requiring three generators for its class group, and related theory," Sierpiński Memorial Volume, Acta Arith., 1972, pp. 71-87.

6. E. S. Golod \& I. R. Safarevič, "On class field towers," Izv. Akad. Nauk SSSR, v. 28, 1964, pp. 261-272. (Russian)

7. Peter Roquette, "On class field towers," in Algebraic Number Theory, Thompson, Washington, D.C., 1967. 